\title{
Book Reviews Comptes rendus
}

François d'Amboise. Dialogues et Devis des Damoiselles, éd. Daniela Costa. Paris, Société des Textes Français Modernes, 1998. Pp. xl, 316.

Le Dialogo de la bella creanza de le donne (1538) d'Alessandro Piccolomini connut pas moins de trois adaptations en français dans la décennie 1572-1581. Daniela Costa a pris le parti de procurer l'édition critique de la troisième adaptation, les Dialogues et Devis des Damoiselles, publiés en 1581 et réédités en 1583 sous le pseudonyme de Thierry de Timphile (François d'Amboisé). L'établissement du texte se signale par sa qualité, car non seulement l'éditeur a relevé en bas de page les variantes des deux éditions des Dialogues, mais elle a en outre souligné les principaux écarts entre, d'une part, le texte source, la première traduction, l'Instruction pour les jeunes dames (1572), proche de l'original italien, et d'autre part, la seconde traduction, adaptation libre intitulée Notable discours (1577) que d'Amboise a largement décalquée. De plus, les rapprochements textuels, les notes d'érudition et le rappel des traités d'éducation féminine sont particulièrement éclairants et bien documentés.

Cependant, l'introduction se révèle nettement moins satisfaisante, parce qu'elle s'appesantit trop sur la prosopographie des relations du traducteur, qui aurait mieux convenu à une notice de dictionnaire, même s'il est vrai qu'elle recèle plusieurs faits inconnus jusque-là, et qu'elle fait l'impasse sur les raisons de la multiplication des traductions françaises de cette décennie. Il demeure que la distinction que l'éditeur opère entre la réception "érotique" (Instruction pour les jeunes dames) et la réception "mondaine" (Dialogues et Notables discours) résume bien la fortune du texte de Piccolomini en France. Pourtant, elle ne tient pas suffisamment compte du contexte et de la proximité temporelle des trois adaptations successives. Il n'est pas indifférent que, au moment où, à la faveur de la Saint-Barthélémy, la réception de Machiavel culmine en France, l'Instruction pour les jeunes dames propose, certes sur le mode ludique, une éducation machiavé- 
lienne, en celà fidèle au Dialogo, contre-modèle à la fois de la donna di palazzo et de la femme chrétienne. De ce point de vue, le Notable discours et les Dialogues et Devis apparaissent comme une véritable gageure, puisqu'ils cherchent à aplanir, tant sur le plan didactique que sur celui du style, le caractère subversif du texte de Piccolomini, que Marie de Romieu, la première traductrice, avait rendu intégralement, pour ramener le dialogue vers l'orthodoxie néoplatonicienne.

D'ailleurs, il est manifeste que les deux adaptations postérieures avaient pour origine la première traduction française, puisqu'elles en reprennent intégralement certains passages et que François d'Amboise va jusqu'à reproduire le sonnet liminaire original. C'est dire dans quelle mesure le Notable Discours et les Dialogues ont été produits en réaction à l'Instruction. L'éditrice, du reste, met bien l'accent sur le fait que l'adaptation de François d'Amboise, par sa table et ses manchettes, voulait s'imposer comme un traité de civilité, bien loin donc du dialogue divertissant et paradoxal du Dialogo, selon l'aveu même de Piccolomini. Elle montre également que cette réécriture procède de la contamination de l'Institution morale du même Piccolomini et du Livre du courtisan et prouve par le fait même l'attachement persistant de la cour de Henri III au modèle de la donna di palazzo de Castiglione, car, en effet, la dédicataire de l'adaptation de François d'Amboise n'est autre que Anne d'Acquaviva, dame d'honneur de Catherine de Médicis. On saura gré à Daniela Costa de nous avoir rendu accessible, avec beaucoup de rigueur philologique et en dépit des quelques lacunes dans la présentation, un texte aussi riche tant sur le plan littéraire, stylistique et rhétorique que du point de vue de l'histoire des mentalités.

\section{CLAUDE LA CHARITÉ, Université de Paris-Sorbonne}

James S. Amelang. The Flight of Icarus: Artisan Autobiography in Early Modern Europe. Stanford: Stanford University Press, 1998. Pp. 497.

Autobiography has obtained a remarkably strong profile in recent scholarship, including the "new cultural history" and literary scholarship which interests itself in the recovery of marginalized voices. That interest, however, has not been accompanied by a sufficient consideration of the historical character of autobiography as a genre. Too often, non-elite autobiography has been implicitly received as an unmediated expression of subjectivity, in effect reproducing earlier scholarship which dismissed such texts as artless. James S. Amelang's new book thus provides an important addition to the scholarship in these areas by examining a broad range of early modern artisan autobiographies in both generic and historical terms.

Amelang has read widely in the available artisan autobiographies of early modern Western Europe and even the American colonies. (An index provides an 